\title{
BMJ Open Vulnerability for new episodes in recurrent major depressive disorder: protocol for the longitudinal DELTA-neuroimaging cohort study
}

Roel J T Mocking, ${ }^{1}$ Caroline A Figueroa, ${ }^{1}$ Maria M Rive, ${ }^{1}$ Hanneke Geugies, ${ }^{2,3}$ Michelle N Servaas, ${ }^{2,3}$ Johanna Assies, ${ }^{1}$ Maarten W J Koeter, ${ }^{1}$ Frédéric M Vaz, ${ }^{4}$ Marieke Wichers, ${ }^{5}$ Jan P van Straalen, ${ }^{6}$ Rudi de Raedt, ${ }^{7}$ Claudi L H Bockting, ${ }^{8,9}$ Catherine J Harmer, ${ }^{10}$ Aart H Schene, ${ }^{1,11,12}$ Henricus G Ruhé ${ }^{1,2,3,5}$

To cite: Mocking RJT, Figueroa CA, Rive MM, et al. Vulnerability for new episodes in recurrent major depressive disorder: protocol for the longitudinal DELTA-neuroimaging cohort study. BMJ Open 2016;6: e009510. doi:10.1136/ bmjopen-2015-009510

- Prepublication history and additional material is available. To view please visit the journal (http://dx.doi.org/ 10.1136/bmjopen-2015009510).

Received 24 July 2015 Revised 20 November 2015 Accepted 6 January 2016

CrossMark

For numbered affiliations see end of article.

Correspondence to

Roel J T Mocking;

R.J.Mocking@AMC.UvA.nl

\section{ABSTRACT}

Introduction: Major depressive disorder (MDD) is widely prevalent and severely disabling, mainly due to its recurrent nature. $A$ better understanding of the mechanisms underlying MDD-recurrence may help to identify high-risk patients and to improve the preventive treatment they need. MDD-recurrence has been considered from various levels of perspective including symptomatology, affective neuropsychology, brain circuitry and endocrinology/metabolism.

However, MDD-recurrence understanding is limited, because these perspectives have been studied mainly in isolation, cross-sectionally in depressed patients. Therefore, we aim at improving MDD-recurrence understanding by studying these four selected perspectives in combination and prospectively during remission.

Methods and analysis: In a cohort design, we will include 60 remitted, unipolar, unmedicated, recurrent MDD-participants (35-65 years) with $\geq 2$ MDDepisodes. At baseline, we will compare the MDDparticipants with 40 matched controls. Subsequently, we will follow-up the MDD-participants for 2.5 years while monitoring recurrences. We will invite participants with a recurrence to repeat baseline measurements, together with matched remitted MDDparticipants. Measurements include questionnaires, sad mood-induction, lifestyle/diet, 3 T structural (T1weighted and diffusion tensor imaging) and bloodoxygen-level-dependent functional MRI (fMRI) and MR-spectroscopy. fMRI focusses on resting state, reward/aversive-related learning and emotion regulation. With affective neuropsychological tasks we will test emotional processing. Moreover, we will assess endocrinology (salivary hypothalamic-pituitaryadrenal-axis cortisol and dehydroepiandrosteronesulfate) and metabolism (metabolomics including polyunsaturated fatty acids), and store blood for, for example, inflammation analyses, genomics and proteomics. Finally, we will perform repeated momentary daily assessments using experience sampling methods at baseline. We will integrate measures to test: (1) differences between

\section{Strengths and limitations of this study}

- Strict and specific inclusion-criteria, matchingprocedure and recruitment-procedure, leading to maximal contrast for major depressive disorder (MDD)-vulnerability, without distortion due to important confounders: MDD-residual symptoms and medication.

- Unique integration of a wide range of measures in a prospective repeated measures design will allow disentangling of recurrent MDD statefactors and trait-factors.

- The extensive assessment procedure needed to measure all variables of interest and confounders will potentially lead to inclusion of participants that are intrinsically aware of the necessity to perform clinical research and readily willing to cooperate.

- Only including participants who currently do not use psychotropic drugs may lead to selection of particular patient subgroups that for example, previously experienced little benefit or adverse effects from medication.

MDD-participants and controls; (2) associations of baseline measures with retro/prospective recurrencerates; and (3) repeated measures changes during follow-up recurrence. This data set will allow us to study different predictors of recurrence in combination.

Ethics and dissemination: The local ethics committee approved this study (AMC-METC-Nr.:11/ 050). We will submit results for publication in peerreviewed journals and presentation at (inter)national scientific meetings.

Trial registration number: NTR3768.

\section{INTRODUCTION}

\section{Rationale}

Major depressive disorder (MDD) is a widespread and disabling mental disorder, with 
estimated worldwide prevalences of $4.3 \%$ annually and $11.1-14.6 \%$ during lifetime. ${ }^{1-4}$ Currently, MDD has the highest burden of any disorder in high-income countries, and is expected to have the second-highest burden worldwide in 2030. ${ }^{5}$ MDD's (in) direct annual excess costs constitute approximately $1 \%$ of the gross domestic product in these countries. ${ }^{6-8}$ Next to suicide and cardiovascular comorbidity, ${ }^{9}$ an important reason for MDD's burden is its recurrent course, ${ }^{2}$ as already indicated by Kraepelin et $a l^{10}$ and formulated by Angst et $a l:{ }^{11}$ 'Single episodes are extremely rare if the period of observation is significantly extended'.

The incidence of recurrences ${ }^{i}$ varies depending on study-characteristics. ${ }^{12-15}$ While recurrent $\mathrm{MDD}$ has been considered as a distinct disease entity (more familiar to bipolar disorder), population studies show that recurrence is widespread in MDD with $\geq 40-75 \%$ lifetime recurrence in patients recovered from a first depressive episode ${ }^{16-19}$ with even higher rates in clinical samples. $^{20} 21$ Our 10-year follow-up study of a specific cohort of recurrent MDD-patients showed an overall $90.3 \%$ recurrence-rate, ${ }^{22}$ with patients being in a depressed state during $13 \%$ of the follow-up time.

During lifetime, MDD-patients are estimated to experience on average about five MDD-episodes. ${ }^{1}{ }^{21}$ Therefore, high recurrence rates pose a major health problem. However, depressive episodes seem to cluster in subpopulations. This also suggests that the most MDD-episodes occur in a relatively limited number of patients. Consequently, if we could lower recurrence rates in these recurring cases, we may greatly reduce the overall number of MDD-episodes and thereby MDD's burden. ${ }^{23}$ If we could a-priori identify these patients at high risk for recurrence, this would provide excellent opportunities for specific, indicated, (secondary) prevention.

For recurrence prevention, antidepressants are most often used, ${ }^{12}{ }^{24}$ but unwillingness to take antidepressants, non-adherence and discontinuation due to adverse effects limit their applicability. ${ }^{25-27}$ As an alternative, preventive cognitive psychotherapies have been developed (eg, mindfulness based cognitive therapy, preventive cognitive therapy and well-being cognitive therapy), ${ }^{28-36}$ which seem to produce long-lasting beneficial effects. ${ }^{22}{ }^{37}$ Nevertheless, recurrence-rates stay substantial, urgently calling for further improvements of recurrence preventing therapy.

In that respect, if we better understand the mechanisms underlying vulnerability for recurrence in MDD, we could (1) use their indicators as (bio)markers to monitor/predict recurrence risk, and/or (2) use these mechanisms to identify/develop novel targets for

${ }^{\mathrm{i}}$ The terms relapse and recurrence are used in the literature and defined as new MDD-episodes within or after $6 \mathrm{mths}$ recovery, respectively. However, empirically there is no clear evidence for this distinction. We hereafter will name both recurrence for clarity. improved and personalised preventive therapy in a precision medicine setting. This early identification and stratified treatment of recurrence risk $^{38}$ could potentially reduce recurrent MDD's disease burden.

However, understanding of mechanisms underlying MDD-recurrence is limited to date. Although remitted MDD-patients have already been studied for a number of years, ${ }^{21}$ most studies investigate MDD during the acute phase. However, to be able to differentiate between trait factors (that remain present during remission and possibly constitute vulnerability for recurrence) versus state factors (which are only present during an MDD-episode), it is necessary to study patients during remission. In addition, the actual predictive associations of these possible trait factors with recurrence have to be tested in long-term prospective follow-ups.

Thus far, the limited research that applied such a prospective approach in remitted MDD-participants investigated several factors as predictive of recurrence. While associated with MDD onset, demographics (eg, gender) generally do not predict recurrence; clinical and social factors seem to be more predictive. Regarding clinical factors, the number of previous episodes is among the strongest predictors, ${ }^{39}$ together with residual depressive symptoms. ${ }^{19}$ In addition, MDD family history, comorbid axis I disorders, age of onset and last episode duration and severity have been suggested as predictors. ${ }^{15} 1940-46$ Furthermore, personality characteristics (coping style and personality traits) and social factors (experiencing daily hassles) have been found to be predictive although findings remain largely inconsistent. In addition, in our previous study a $71 \%$ variance in time to 5.5 years recurrence remained unexplained, ${ }^{47} 48$ and only few actual predictive factors were potentially modifiable.

As indicated, the pathophysiology behind these factors' predictive properties for recurrence remains far from understood. For example, residual symptoms predict recurrence in a short-term interval but seem less predictive in the long-term interval. ${ }^{49}$ This indicates that residual symptoms may not constitute a vulnerability trait, but rather reflect the early initiation of a new episode or an earlier episode not yet in full remission. In addition, the predictive effect of previous episodes can be explained due to scarring (increasing vulnerability directly resulting from experiencing previous episodes) or high premorbid vulnerability (pre-existing abnormalities leading to previous episodes and new recurrences). ${ }^{50-52}$ From the prediction perspective, these pathogenetic differences might seem a merely academic question. However, identifying the mechanisms underlying MDD-recurrence is essential to discover better potential targets for innovative preventive interventions to increase resilience.

\section{Study aims and outline}

Based on the above, the present study aims at advancing the knowledge on (1) factors that are associated with 
recurrent MDD-vulnerability, (2) how these factors are related with each other, (3) their predictive association with prospective recurrence and (4) their change during recurrence.

In order to do so, we will initially compare fully remitted unmedicated recurrent MDD-participants to matched healthy controls. Subsequently, we will monitor recurrence(s) in the MDD-participants during a 2.5-year follow-up and repeat measurements when an MDD-participant experiences a recurrence during follow-up. Below, we will first outline our theoretical framework to provide background for our hypotheses regarding the specifically selected factors that we will investigate.

\section{Theoretical framework}

Based on preliminary findings, theoretical literature, and observations from adjacent fields, several theories have been developed to explain recurrence pathogenesis. Here, using a stratified approach, we aim to introduce and integrate theories from four distinct selected levels of perspective: ${ }^{53} 54$ symptomatology, affective neuropsychology, brain circuits and endocrinology/ metabolism (figure 1).

\section{Symptom level}

A disturbed balance between negative and positive valence systems seems to lie at the heart of MDD symptomatology. ${ }^{54}$ Regarding negative valence systems, MDD-patients suffer from for example, negative affect, rumination and dysfunctional cognitions. While negative cognition and processing styles as rumination usually resolve after remission, they may remain present in latent form, and can be reactivated during (mild) dysphoria, which is conceptualised as "cognitive reactivity'. 5056 Interestingly, latent dysfunctional attitudes, increased cognitive reactivity and rumination have all been found to predict recurrence in remitted MDD-participants. ${ }^{57} 58$ Relating to negative but also positive valence systems, anhedonia (inability to experience pleasure) is one of MDD's core symptoms. Apart from the ability to experience joy, the rewarding effect of pleasure can also have a motivational function: pleasurable events appear to reinforce behaviour leading to these events (conditioning). This implies that experiencing pleasure is a necessary stimulation to learn associations between stimuli and (pleasurable) outcomes and move an individual to perform certain behaviours. MDD-patients have difficulties in experiencing the rewarding effects of positive/pleasurable events when depressed, particularly relative to aversive stimuli, and indeed have difficulties learning new beneficial behaviours. This can also be observed in the form of psychomotor retardation and decreased positive affect. However, anhedonia remains relatively underinvestigated during remission, and it remains largely unknown to what extent anhedonia can predict recurrence (see for reviews ${ }^{59-63}$ ).

\section{Affective neuropsychological level}

This disturbed balance between negative and positive valence systems at the symptom level may relate to negative biases in emotional processing at the affective ('hot') neuropsychological level. Negative biases manifest themselves when (dis)engaging (ie, attentional bias), memorising, error-monitoring, shifting attention between or regulating emotional information. ${ }^{64-74}$ Negative biases are thought to result from increased negative attention on the self, and are thus related with negative self-referential processing styles as rumination and cognitive reactivity, which show a reciprocally reinforcing relationship with negative affect. $^{75} \quad 76$ Increasing evidence shows that negative self-referential processing and associated brain alterations contribute greatly to the course and development of MDD. ${ }^{75}$ With respect to reward processing, negative biases manifest in decreased reward sensitivity (negative valence) and increased aversive stimulus sensitivity (positive valence) ${ }^{54}$ However, the precise relations between these concepts, and to what extent these negative emotional processing biases with associated brain alterations remain present during remission, and can predict recurrence, remains largely unknown. ${ }^{77} 78-80$

\section{Brain circuit level}

From a neurobiological brain circuit perspective, disturbed emotional processing at the affective neuropsychological level may be observed as an imbalance between emotional (limbic/ventral) and regulating (cognitive/dorsal) regions. ${ }^{81-86}$ Specifically, emotional brain regions seem hyperactive in response to negative stimuli but hypoactive to positive. ${ }^{87}$ In addition, regulating regions are generally hypoactive but may show compensatory hyperactivity under certain circumstances, for example, more automatic emotion regulation. ${ }^{88}$ This may be explained by altered functional and structural connectivity between these regions. ${ }^{89}$ Furthermore, disturbed functioning of the default-mode network, a network that is involved in self-referential processing and is negatively correlated to regions that process attention and cognitive control, has consistently been observed in MDD. ${ }^{90-94}$ Aberrations in the default-mode network (ie, failure to deactivate default mode network $(\mathrm{DMN})$ regions) ${ }^{95}$ during tasks as well as DMN hyperconnectivity ${ }^{96}$ during rest have been observed in MDD. DMN aberrations have been associated with emotionalcognitive disturbances and increased negative self-focus, such as rumination. ${ }^{58} 759497-106$

Especially anhedonic MDD-patients have a decreased ability to change behaviour in relation to rewards, which appears to persist after remission. ${ }^{59} 107$ This reduced reward responsiveness might be related to blunted phasic dopaminergic signalling. Indeed, reinforcement learning appeared impaired in depressed MDD-patients versus controls, with blunted reward signals in the ventral striatum, and increased compensatory ventral tegmental area activations when thirsty patients were 

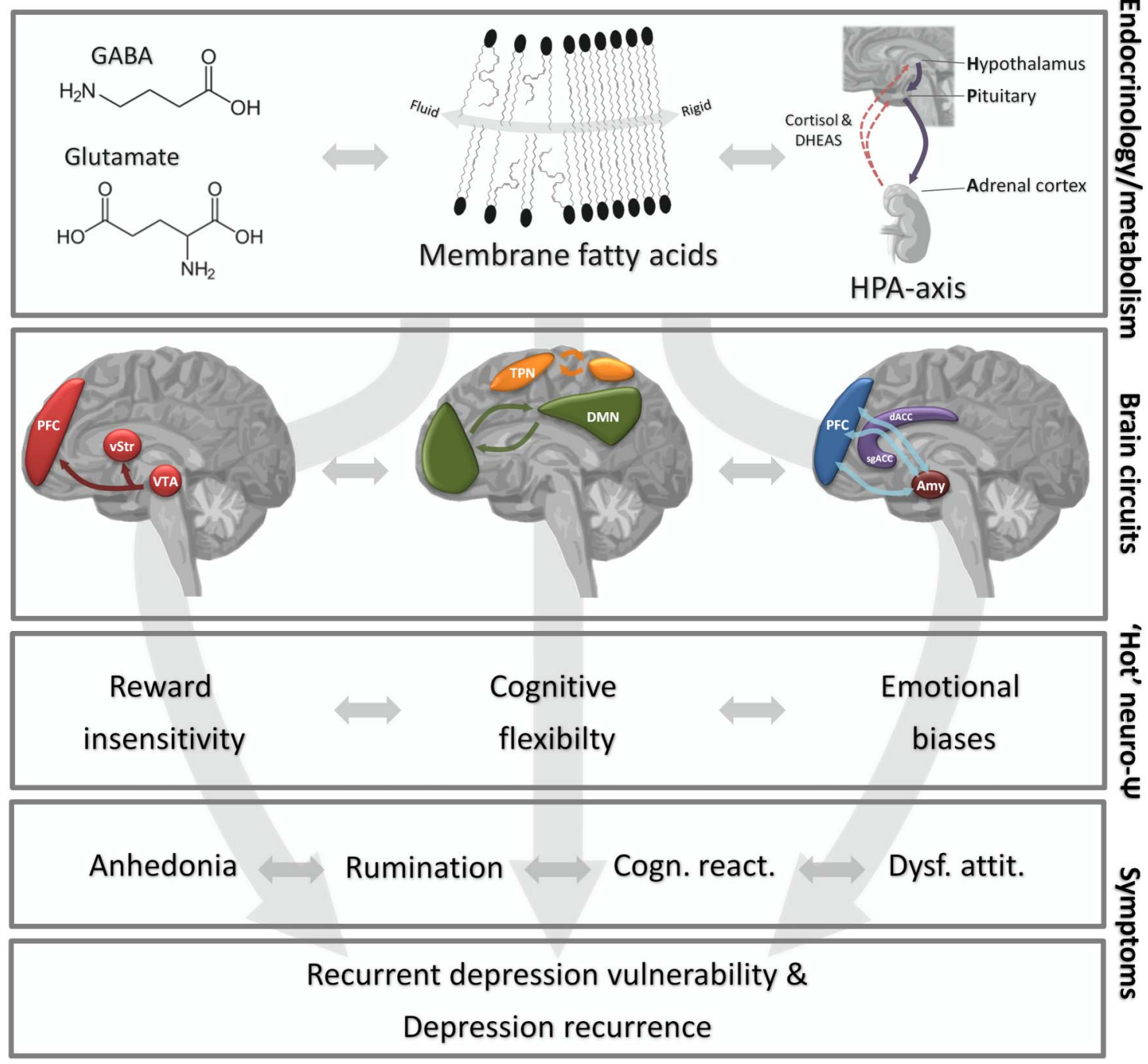

Figure 1 Theoretical framework. Schematic representation of the theoretical framework of the present DELTA-neuroimaging study. The four selected levels of perspective (endocrinology/metabolism, brain circuits, affective neuropsycholoy and symptoms), their respective subdomains, and their connections have been depicted. The horizontal straight arrows show potential bidirectional relationships (for readability bidirectional relationships between eg, anhedonia and cognitive reactivity are not shown), the horizontal curved arrow shows membrane fluidity balance, coloured arrows show potential connections, dashed arrows show inhibiting effects and vertical grey arrows show possible underlying pathways. Abbreviations used: DELTA, Depression Evaluation Longitudinal Therapy Assessment; DHEAS, dehydroepiandrosterone-sulfate; GABA, $\gamma$-aminobutyric acid; HPA, hypothalamic-pituitary-adrenal; PFC, prefrontal cortex; vStr, ventral striatum; VTA, ventral tegmental area; TPN, task positive network; DMN, default mode network; dACC, dorsal anterior cingulate cortex; pgACC, pregenual anterior cingulate cortex; Amy, amygdala; 'Hot' neuro- $\Psi$, affective neuropsychology; Cogn. react., cognitive reactivity; Dysf. attit., dysfunctional attitudes.

learning associations between stimuli and water delivery. ${ }^{108}$ Furthermore, MDD-patients show reduced reward anticipation and are less prone to exert effort for a potential reward. ${ }^{59}$ These abnormalities also appear present in participants prone to develop MDD. ${ }^{109}$ Also, recognition of reward-related stimuli appeared most difficult and associated with most impaired brain activities in the N. accumbens, anterior cingulate cortex (ACC) and ventromedial prefrontal cortex in patients with chronic recurrent MDD. ${ }^{110}$ Thus, dopaminergic reward-related brain circuits seem to be of importance in recurrence of MDD. However it remains unclear whether such abnormalities in reward-related learning are also associated with recurrence.
Despite increasing research efforts to delineate these brain circuits, it is hardly investigated how the defaultmode network and its relations to other cognitive networks and emotion-processing and reward circuits function in remitted recurrent MDD-participants. ${ }^{111-116}$ In addition, it has been examined scarcely how alterations in these circuits can predict recurrence in remitted MDD-participants. ${ }^{117} 118$

\section{Endocrinology and metabolism}

These disturbed brain circuits may be associated with alterations in endocrinology and metabolism. From an endocrinological viewpoint, the principal stress systemthe hypothalamic-pituitary-adrenal (HPA)-axis-has 
been studied extensively in MDD. ${ }^{119}$ In combination with, for example, findings in first degree relatives, our own research indicates that HPA-axis hyperactivity is an endophenotypic trait, with higher diurnal cortisol and altered dehydroepiandrosterone-sulfate (DHEAS) that remain during remission, ${ }^{21} 120121$ and potentially predict recurrence. ${ }^{122-125}$ Interestingly, HPA-axis activity can be linked with brain circuit alterations. For example, the effects of stress on limbic network structure in MDD could reflect chronic HPA-axis hyperactivation-induced allostatic load (eg, reducing hippocampal volumes), predisposing to MDD(-recurrences). ${ }^{126} 127$ Vice versa, the HPA-axis is controlled by the limbic system, ${ }^{128}$ through medial prefrontal connections with amygdala and hypothalamus. ${ }^{129}$

Moreover, interestingly, we previously showed a bidirectional relationship between fatty acid metabolism and HPA-axis activity. ${ }^{130} 131$ Fatty acids are main constituents of (nerve) cell membranes and myelin, and so influence important (neuro)physiological mechanisms such as exocytosis, membrane-anchored protein function, membrane fluidity, second messenger system activity and white matter integrity. ${ }^{132} 133$ Furthermore, they are precursors of eicosanoids and are associated with brainderived neurotrophic factor, which regulate inflammatory homeostasis and nervous system architecture, respectively. ${ }^{9}{ }^{133-136}$ We previously showed that besides alterations in $\omega-3$ fatty acids, MDD is additionally associated with more general alterations in overall fatty acid metabolism, also in recurrent MDD. ${ }^{137-140}$ However, inconsistencies remain, and recurrent MDD has only been sparsely investigated. Moreover, given the widespread involvement of fatty acid metabolism in brain physiology, associations between fatty acid metabolism and brain circuit alterations can be expected, ${ }^{9} 136141$ but remained largely uninvestigated thus far.

Furthermore, glutamate/glutamine and $\gamma$-aminobutyric acid (GABA) neurometabolism is currently considered an interesting additional system in MDD and its recurrence too. Glutamate and GABA are the major excitatory and inhibitory neurotransmitter, respectively, and have been implicated in MDD-pathophysiology. ${ }^{142}{ }^{143}$ For instance in depressed MDD-patients, excess excitotoxic synaptic glutamate have been suggested to cause less pregenual ACC deactivation when viewing negative emotional pictures. ${ }^{144145}$ Nevertheless, previous investigations of glutamate/GABA in depressed MDD-patients remain contradictory, ${ }^{146} 147$ and while abnormalities might normalise after remission, ${ }^{148}$ this is only sparsely investigated, ${ }^{147} 149$ especially not in recurrent MDD.

\section{Summary of theoretical framework}

MDD can be characterised by multiple alterations across systems that remained distinct thus far, but potentially can be integrated. At the symptom level, MDD-patients show a disturbed balance between negative and positive valence systems with increased latent negative affect, rumination, dysfunctional cognitions and cognitive reactivity, together with anhedonia. This may be associated with negative emotional biases at the affective neuropsychological level. These negative emotional biases may relate to an imbalance between emotional and regulatory brain circuits, DMN hyperconnectivity/ activity and might also be associated with a disturbed brain reward circuit. These brain circuit alterations seem closely connected with HPA-axis alterations, which seem bidirectionally related with fatty acid and glutamate/ GABA-metabolism (figure 1). However, even if previous research studied remitted MDD-participants, these alterations were mostly investigated in isolation and only crosssectionally. Consequently, it remains largely unknown to what extent these alterations (1) persist during remission, (2) are associated with each other, (3) are predictive for recurrence and (4) change during recurrence.

\section{Hypotheses}

With the aim of the current 'DELTA-neuroimaging' study to integrate these factors and test their association with recurrence in a prospective cohort-study of stably remitted unmedicated recurrent MDD-participants, we first will compare MDD-participants with carefully matched controls at baseline, and subsequently we will follow-up the MDD-participants for 2.5 years while monitoring recurrences. Moreover, we will invite recurring participants to repeat baseline measurements, together with matched remitted participants. Following this line of research we will investigate the following specific hypotheses:

1. Compared to matched never-depressed controls, remitted unmedicated recurrent MDD-participants will show (ie, a trait effect):

A. At the symptom level, a disturbed balance between negative and positive valence systems with increased rumination, dysfunctional cognitions, cognitive reactivity and anhedonia.

B. At the affective neuropsychological level, increased negative biases in emotional processing when (dis)engaging (attentional bias), memorising, shifting attention between, and regulating emotionally valenced stimuli.

C. At the brain circuit level, altered grey/white matter structure and function/connectivity of emotional/regulating regions, reward brain circuits and the default-mode network, also relative to other networks of the brain, with specifically:

i. More ventral and less dorsal region activation when viewing emotional pictures.

ii. Less connectivity between ventral and dorsal regions.

iii. More activation of dorsal regions during a reappraisal emotion regulation task.

iv. Blunted ventral striatum and increased ventral tegmental area reward-signals.

v. Hyperconnectivity within and dominance of the default-mode network at rest, which becomes more pronounced after sad mood-induction. 
D. At the endocrinology and metabolism level, altered HPA-axis activity, fatty acid metabolism and emotional network GABA/glutamate, with:

i. Higher morning and evening HPA-axis cortisol and relatively lower DHEAS, which becomes more pronounced after sad mood-induction.

ii. Lower degree of fatty acid unsaturation, chain length, peroxidisability and $\omega-3 /$ $\omega$-6-ratio.

iii. More glutamate and less glutamine/GABA signals in the basal ganglia and pgACC, which becomes more pronounced during sad mood-induction.

2. In remitted unmedicated recurrent MDDparticipants the above systems will be related with clinical characteristics (number of previous episodes, residual symptoms and age of onset) and each other, and these latter mutual relationships will differ from those in matched never-depressed controls.

3. In remitted unmedicated recurrent MDDparticipants, above alterations will predict prospective 2.5-year follow-up symptom course, specifically:

A. Time until recurrence

B. Cumulative number and severity of MDD-episodes

C. Course of depressive (residual) symptoms.

4. The above alterations will become more pronounced during repeated measures in recurrent MDDparticipants experiencing a recurrence during follow-up, in comparison to repeated measures in matched remitted recurrent MDD-participants (ie, a state effect).

\section{METHODS}

\section{Design}

The present study consists of two stages (figure 2). First, using a cross-sectional patient-control design, we will compare remitted recurrent MDD-patients with matched never-depressed controls, to identify traits that remain present during remission and that are associated with recurrent MDD-vulnerability. Second, using a prospective cohort-design, we will follow-up the patients. During follow-up, we will measure depression symptoms every 4 months, to see whether we can predict clinical course from baseline measures. Moreover, when we detect a follow-up recurrence, we will invite the respective patient to repeat several baseline measures. In addition, we will invite remitted patients (matched on duration of follow-up, gender, age, educational level and working class) to repeat the measures as well. While this repeated measures design is not required to predict recurrence, it is of interest as it allows us to identify depression state versus trait-effects.

In sum, we will first test for trait factors associated with MDD-vulnerability by contrasting vulnerable (remitted recurrent MDD) versus resilient (never-depressed controls) participants. Subsequently, also in order to further delineate whether these identified factors are causal, consequences or confounders, we will test their predictive effect of prospective recurrence during follow-up in the remitted recurrent MDD group. Finally, we aim at disentangling state and trait effects by repeating measures in patients during recurrence versus matched patients who are in current remission. Below, we will describe the population, measures, procedure and analyses in detail

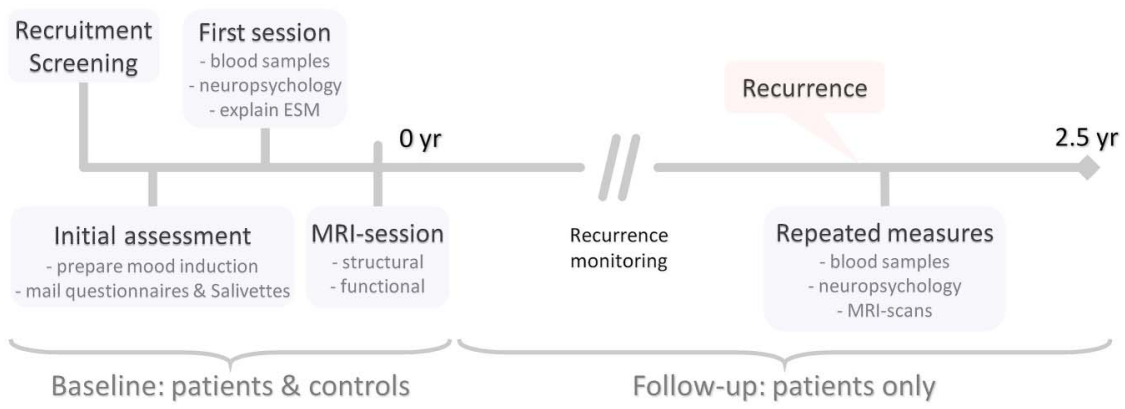

Figure 2 Study design. Figure 2 depicts the study design of the present Depression Evaluation Longitudinal Therapy Assessment (DELTA)-neuroimaging study. Different part of the study are shown in chronological order from left to right. For a description of the contents of questionnaire booklets and tasks we refer to the online supplementary text. After screening, recruited patients and controls participate in the initial assessment where we check inclusion and exclusion criteria, register variables and covariates of interest, prepare the mood induction and mail questionnaire booklet I and Salivettes. During the subsequent first study session we will take fasting blood samples, perform the affective neuropsychological tests, perform the sad mood-induction, explain the experience sampling method (ESM) and the emotion regulation functional MRI (fMRI) task, and hand out the ESM-psymate and questionnaire booklet II. Subsequently, participants come to the MRI-session, where we take structural (T1-weighted and diffuse tensor imaging (DTI)) and fMRI-scans (neural and sad mood induction resting state, reinforcement learning, cued emotional conflict, emotion regulation), as well as $\gamma$-aminobutyric acid (GABA)-edited MR spectroscopy (MRS) of the basal ganglia and pregenual anterior cingulate cortex. Next, we monitor the patients by calling them every $\sim 4$ months to assess recurrence. In case we detect a recurrence, we invite the respective patient-together with matched non-recurrent patients-to repeat part of the baseline assessments (blood samples, affective neuropsychological tests, structural $\mathrm{MRI}, \mathrm{fMRI}$ (resting state, reinforcement learning) and MRS). 
in that order, additional information can be found in the online supplementary tables S1 and S2.

\section{Population}

\section{Inclusion criteria}

To maximise contrast for recurrent MDD-vulnerability, without confounding effects of medication or current MDD-symptoms, we will include recurrent MDD-participants ( $\geq 2$ previous MDD-episodes as assessed using the structured clinical interview for Diagnostic and Statistical Manual of Mental Disorders, fourth edition (DSM-IV) diagnoses (SCID) ${ }^{150}$ who are in stable remission ( $\geq 8$ weeks with a 17-item Hamilton Depression Rating Scale (HDRS) $\leq 7$ and not fulfilling the criteria for a current MDD episode (as assessed using the SCID during inclusion) ). ${ }^{151}$ Specifically, we will include participants aged $35-$ 65 years, to include a homogeneous age group, and to preclude conversion to bipolar disorder due to later experience of (hypo)manic episodes. Of note, despite overall high recurrent MDD vulnerability and homogeneity regarding, for example, age, we expect this group of MDD-patients to exhibit considerable variance in prospective recurrence rates. For example, in our previous research $^{22} 3047152$ the range in previous MDD-episodes was from 2 to 60 , and we will now include patients with none or only a single episode in the past 10 years. We expect that this will lead to a relapse rate of $\pm 50 \%$ during the 2.5 years follow-up, providing excellent within-group contrasts for prospective recurrence in this overall highly vulnerable group. Second, we will include relatively resilient controls without personal (SCID) or first degree familial psychiatric history, carefully matched for age, sex, educational level, working class and ethnicity.

\section{Exclusion criteria}

While comorbidity in general will not be an exclusion criterion because it may be an important predictor, in order to obtain a homogeneous sample we will exclude participants with current diagnoses of alcohol/drug dependence, psychotic or bipolar, predominant anxiety or severe personality disorder (all SCID); standard MRI exclusion criteria (eg, metal objects in the body, claustrophobia); electroconvulsive therapy within 2 months before scanning; history of severe head trauma or neurological disease; severe general physical illness; no Dutch/English proficiency. To minimise inclusion bias, we will try to familiarise mildly claustrophobic participants in a mock MRI-scanner to enable actual MRI-assessments. If this does not succeed, we will only perform non-MRI assessments. All participants have to be without psychoactive drugs/medication for $>4$ weeks before assessments. We will allow incidental benzodiazepine use, but this must be stopped after informed consent and $\geq 2$ days before assessments. Despite possible effects of psychotherapy we will not exclude current or past psychotherapy due to feasibility reasons. However, we will assess all forms of therapy used, report these and treat them as covariates in our analyses.

\section{Recruitment}

To minimise selection biases, we will recruit both groups through identical advertisements in freely available online and house-to-house papers, posters in public spaces and from previous studies in our and affiliated research centres. One previous study from which we will recruit participants is the Depression Evaluation Longitudinal Therapy Assessment (DELTA)-study. ${ }^{30} \mathrm{We}$ recently completed the 10-year follow-up of this randomised controlled trial assessing the protective effects of 8 weeks preventive cognitive therapy on recurrence in recurrent $\mathrm{MDD} .^{22}$ In this long-term study, we not only obtained detailed psychological, but also obtained biological measures, which can be linked to data obtained in the present study in the same participants. Of note, the original DELTA sample was recruited like the procedure for new participants for the present recruitment, among others through newspaper advertisements. By DELTA-study design, $50 \%$ of the original DELTA sample received randomised preventive cognitive therapy 10 years ago, however, as (1) previous psychotherapy was not an exclusion criterion in the present total sample and (2) the preventive cognitive therapy intervention was more widely implemented in the Netherlands since the DELTA-study, non-DELTA participants could also have undergone this treatment. This allows the additional interesting option to collect data on previous treatments in all participants in order to estimate the magnitude of this possible treatment effect. Finally, we will recruit additional recurrent MDD-participants from patients previously treated by the AMC or affiliated general practitioners and psychologists.

\section{Measures}

See online supplementary tables S1 and S2 for full details.

\section{Structured interview and questionnaires}

The SCID is widely accepted as structured diagnostic interview to adequately assess DSM-IV defined psychiatric disorders. ${ }^{150} 153$ Questionnaire-booklets I-IV (see online supplementary text) include questionnaires on depressive symptoms (eg, HDRS), stress and life events (trauma and daily hassles), personality (neuroticism and coping) and lifestyle (physical activity, sleep and diet).

\section{Mood induction}

We will prepare a negative and neutral mood-induction procedure by asking participants to recall and describe a personal sad and neutral memory, ${ }^{56}$ from which we will make sad and neutral personalised scripts. In addition, we will request participants to listen to and rate five different fragments of sad/neutral music on a dedicated website (accessible on request). This type of provocation (combining sad music with autobiographical recall) has been shown to effectively induce transient dysphoric mood states. ${ }^{56}$ We used this mood induction to test (1) mood-induced changes in dysfunctional attitudes 
(cognitive reactivity), (2) HPA-axis activity and (3) brain networks.

\section{Affective neuropsychological tests}

The affective neuropsychological tests all assess emotional processing. The exogenous cueing task allows disentangling of attentional engagement and disengagement components in attentional bias. ${ }^{66} 154$ The facial expression recognition task measures interpretation of key emotionally valenced social signals of varying intensity (morphed faces). ${ }^{69} 71155$ The emotional categorisation task assesses response speed to self-referent positive and negative personality descriptors, the emotional memory task follows up on this task by assessing surprise (free) recollection memory of these personality descriptors. ${ }^{69} 71155$ The internal shift task examines capacity to shift attention between working memory contents in response to emotional and non-emotional material. ${ }^{156} 157$ For matching purposes, we will estimate premorbid intelligence with the Dutch adult reading test. ${ }^{158}$

\section{Experience sampling method}

Momentary assessment techniques are ideal for prospective examination of dynamics of observed behaviour, and enable to capture the film rather than a snapshot of daily life. ${ }^{159-162}$ Experience sampling method (ESM) is a structured diary method developed to study participants in their daily surroundings, applicable via a validated interactive ESM-palmtop. We will obtain ESM-ratings regarding positive and negative affect-hypothesised to be separate but correlated latent factors ${ }^{163}$-and possible influencing factors (e.g. (social) activities), for 6 days with 10 semirandom measurements/day preferably between the first study-session and MRI-session.

\section{MRI-scans (two blocks)}

In the first block, after locater and reference scans, a structural T1-scan will provide high resolution threedimensional anatomical information. Then we will obtain a resting-state scan after neutral moodinduction, ${ }^{98}$ followed by a reinforcement learning fMRI-task which applies a Pavlovian-learning paradigm delivering the thirsty participants small amounts of sweet or bitter solution at $80-20 \%$ probabilities after conditional stimuli. This enables assessment of reinforcement learning circuitry. ${ }^{108}$ Subsequently, using a GABAspecific MEGA-PRESS sequence we will obtain an edited ${ }^{1} \mathrm{H}$ J-difference MR spectroscopy (MRS)-scan of the basal ganglia to measure glutamate and GABA. ${ }^{144}$ 164-166 A diffusion-weighted imaging (DTI) spin echo sequence will assess white matter structure. ${ }^{167}$ After a break, in the second block, participants will perform the cued emotional conflict fMRI-task, which will test cue related conflict anticipation and response related cognitive control. ${ }^{168}$ Then, the emotion regulation task will measure brain activity in emotional and regulatory brain networks during attending and regulating (distancing technique) positive, negative and neutral emotional stimuli. Subsequently, we will make another resting-state scan, but this time after a negative mood-induction. In combination with the neutral resting-state scan from the first block, this sad mood-induced resting-state scan will allow assessment of mood-induced changes in brain network interactions. ${ }^{98}$ Finally, we will make another MRS-scan of the pgACC. In the follow-up MRI-scansession we will repeat the structural, resting state (without mood-induction), reinforcement learning and MRS-scans. During scanning we will record heartbeat and breathing in order to correct for their movement-effects.

\section{Blood measures}

From collected blood tubes, we will use $1 \times 4.5 \mathrm{~mL}$ EDTA blood for fatty acid analyses in washed erythrocytes (as a model of neuronal membranes) ${ }^{139}$ which we will store for future lipidomic analyses. We will use $7 \mathrm{~mL}$ EDTA and PaxGene blood collection tubes for future genomic analyses (eg, serotonin, dopamine, glutamate/GABAcascades, one-carbon metabolism or HPA-axis receptors). ${ }^{38} 169170$ We will store platelet-poor plasma from $5 \mathrm{~mL}$ citrate blood and also store plasma from $4.5 \mathrm{~mL}$ EDTA and lithium-heparine blood collection tubes for future use (eg, metabolomics and inflammation). ${ }^{171}$

\section{Salivary measures}

As described below and in the online supplementary text, we will instruct participants to collect salivary samples over the day using Salivettes (Sarstedt, Nümbrecht, Germany). Saliva reflects blood cortisol and DHEAS-concentrations, but enables minimally intrusive and relatively stress free assessment. ${ }^{120} 172173$

\section{Procedure}

We will regularly train all assessors and experienced psychiatrists will closely supervise the assessment procedures. We will discuss difficult assessments; in case of disagreement we will make a conservative decision (eg, exclusion)

\section{Preparation}

\section{Initial assessment and mood-induction}

We will telephonically screen recruited participants for potential eligibility. In a first interview (telephonically or face-to-face), we will check inclusion and exclusion criteria. After obtaining informed consent we will register psychiatric and somatic treatment history, covariates of interest and potential confounders. Furthermore, we will mail questionnaire-booklet I (see online supplementary text) and Salivettes, with detailed instructions. In addition, we will prepare the mood-induction procedure.

\section{Baseline visits}

\section{First study-session}

We will instruct participants to arrive after $\geq 8 \mathrm{~h}$ fasting. First, we will collect blood samples by venipuncture, which we will directly bring to the laboratories. 
Subsequently, we will allow participants to eat and drink, with the exception of caffeinated drinks.

Next, we will instruct participants to perform the neuropsychological tests in two blocks with a break in between, and measure waist circumference ${ }^{140}$ (see online supplementary text). After neuropsychological testing, we will explain the scanning procedure and train the participant for the emotion regulation fMRI-task, which will be performed in the scanner (see online supplementary table S2). After a 15-min break, participants will undergo the sad mood-induction. Before and directly after sad mood-induction, we will request participants to fill out a Dysfunctional Attitudes Scale (two randomised counterbalanced versions), ${ }^{56} 174 \quad 175$ rate their sadness on a visual analogue scale and collect saliva (using Salivettes).

Finally, we will explain and instruct participants about the ESM (see above). In addition, we will provide participants with questionnaire-booklet II (see online supplementary text) to fill out before the MRI-session.

\section{MRI-session}

We will instruct participants to arrive thirsty, that is, $\geq 6 \mathrm{~h}$ without drinking and $\geq 2 \mathrm{~h}$ without eating juicy food (for the reward learning task). On a Philips Achieva XT 3 T MRI (Philips Medical Systems, Best, the Netherlands), using a 32-channel receiver headcoil, at the University of Amsterdam, Spinoza Center, we will scan two consecutive blocks of approximately $60 \mathrm{~min}$ each (see above), separated by a break. During the scanning procedure, we will again perform the mood-induction (neutral/sad) in a slightly modified version as described previously. ${ }^{56}$ We will ask participants to listen to their selected most neutral/sad music piece and meanwhile read their personal sad/neutral memories presented on a screen in the scanner (during $5 \mathrm{~min}$ ), directly before the resting state scans. Finally, we will debrief participants, complete questionnaire-booklet III (see online supplementary text), and obtain post-scan ratings of stimuli presented during the tasks.

\section{Follow-up}

\section{Monitoring}

We will follow-up the recurrent MDD-participants by regular (every $\sim 4$ months) phone-calls (SCID and HDRS) and questionnaire-booklet IV (see online supplementary text). To maximise recurrence detection rates, we will also instruct participants to contact us at the moment they subjectively experience a recurrence and inform a person close to them of these instructions.

To allow for the possibility to disentangle state and trait effects, when we detect a recurrence (SCID), we will invite the respective recurring participant and a matched remitted (MDD-participant to repeat several baseline measurements (see below). We will preferably scan participants before they (again) start antidepressants, but-in order to maintain power-this will not be an exclusion criterion for the follow-up scan/ measurements. Thus, when patients experience a relapse and agree to participate in the study again, they will be matched with recurrent MDD-participants that are in remission (SCID and HDRS $\leq 7$ ) and meet matching criteria. We will conduct matching based on grouplevel characteristics of relapse patients versus control patients (mean and distribution of follow-up time, age, years, sex, educational level and working class). In this way, we also aim to include relatively more control patients (relapsed:control patients ratio of 1:1.5), with the goal of increasing power. These matched participants have to be currently euthymic but can have had a prior relapse, thus after the baseline measurement, or a relapse during follow-up after second participation. The reason for this approach is that we are interested in comparing the effect of depression (state) versus depressive vulnerability (trait), instead of simply comparing more vulnerable patients to stable patients. This will give us insight into the pathophysiology of relapse versus remission; it allows to examine which factors stay the same, and which factors show change when patients relapse. Potential in-between recurrences will, however, be examined as a potential confounder in the final analyses. Nevertheless, a participant will not be included more than once in the follow-up repeated measurements (scanning/neuropsychology), in order to exclude the possibility of learning effects and habituation in testing/scanning and prevent complex covariance structures.

\section{Repeated measures in recurring and matched MDD-participants}

We will repeat questionnaire-booklets I-III (see online supplementary text), blood sampling and neuropsychological testing. In addition, we will repeat part of the MRI-scan in an $\sim 1 \mathrm{~h}$ scan-session (see online supplementary table S2). To minimise learning effects, we will use randomised counterbalanced versions of tasks when applicable. We will not repeat the moodinduction.

\section{Statistical analysis plan \\ E-infrastructure and software}

We will store raw and cleaned data on dedicated servers and make use of available e-infrastructure bioinformatics networks where necessary. ${ }^{176}$ We will use a variety of programmes under which SPSS (IBM SPSS, Chicago, Illinois, USA).

\section{Data preparation}

\section{Distributions and missing data}

We will inspect distributions and remove (multivariate) outliers and data noncompliant to the protocol (eg, saliva samples outside time-range or chance level (neuro) psychological responses). We will transform non-normally distributed data where possible, otherwise we will apply non-parametric tests or bootstrapping if applicable. For 
extensive missing data at random, we will use multiple imputation where necessary and possible. ${ }^{139} 177178$

\section{(Neuro)psychological tests}

For the (neuro)psychological tests, we will calculate summation-scores where applicable. ${ }^{71} 155$

\section{ESM}

We will prepare ESM-data using developed algorithms. In brief, we will include data in the analyses for which $>30 \%$ ESM reports are within $25 \mathrm{~min}$ after the programmed time of the beep, ${ }^{179}$ to ensure reliability. ${ }^{180}$ From the ESM-data we will test the factor structure of the positive and negative affect measures using factor analysis, also at the within-participant level (see online supplementary table S1). ${ }^{163}$

\section{MRI-data}

We will perform standard preprocessing using dedicated software. ${ }^{181} 182$ After realignment, we will co-register functional scans to the structural scan, and thereafter normalise to the standard Monteal Neurological Institute (MNI) brain or a DARTEL template (Diffeomorphic Anatomical Registration Through Exponentiated Lie Algebra) for more flexible group normalisation, and smooth. For the different fMRI paradigms, we will perform fixed effect analyses on single-participant level with linear regression techniques (general linear models). For DTI-scans, we will use tract-based spatial statics for general effects and tractography for a priori defined tracts of interest. ${ }^{183}$

\section{Neurometabolism and HPA-axis}

We will quantify glutamate and GABA based on acquired MRS-spectra. ${ }^{165}$ From concentrations of all measured fatty acids, we will calculate overall fatty acid unsaturation, chain length and peroxidisability using dedicated indices. ${ }^{139}$ Finally, we will calculate cortisol/DHEAS-ratio as indication of HPA-axis balance. ${ }^{184}$

\section{Statistical analyses}

The statistical analysis protocol has been written, and the study statistics will be carried out, under close supervision of a statistical specialist.

\section{Power analyses}

Power analyses for continuous and categorical outcomes of the cross-sectional and prospective analyses show adequate power to detect small to medium effect-sizes with 60 patients and 40 controls (see online supplementary text). This is in line with previous comparable research that found significant effects in smaller samples. ${ }^{66}$ Power calculations for studies involving MRI remain hard and are not used routinely (for an approach see eg, Mumford and Nichols, ${ }^{185}$ Hayasaka et $a l^{186}$ and Murphy et $a l^{187}$ ). Currently, there is consensus that groups of $\geq 20$ usually yield sufficient power in MRI-studies to detect moderate differences in regions of interest.
Based on these power estimations and feasibility aspects, we will test our first hypotheses on acquired scans from 60 recurrent MDD-participant and 40 controls, which is for baseline group comparisons a number more than common in MRI studies, also in studies with a comparable design. ${ }^{188}$ Regarding feasibility, next to scanning costs which limit participant number, recruitment efforts were estimated based on our previous studies. These efforts will be manageable with this sample size of specifically remitted recurrent MDDpatients that have to be medication free. ${ }^{30}$

Regarding the prospective analyses, in a previous study with recurrent MDD-participants, we observed $\sim 50 \%$ recurrence rate in 2.5 years. ${ }^{30}$ We therefore expect $2 \times 20-30$ participants to be eligible for a second scan and subsequent comparisons, allowing for some dropouts. Based on previous research in comparable samples we expect low attrition rates. ${ }^{22} 3047152$ Moreover, all participants can be included in the Cox-regression analyses, since these can adequately deal with attrition (outcome measure incorporates time to event or censored end of observation). As not all participants will be identified when the recurrence is present and/or not all recurrent patients will be available for a second scan, we expect to obtain two groups of \pm 20 patients with or without a recurrence up who will be scanned again during follow.

We perform a large set of measurements, which carries the risk of false positives. However, as we will perform analyses according to analysis-plans which are a priori specified, we will do so for independent a priori hypotheses. In addition, we will use multivariate analysis techniques (eg, machine-learning) to further reduce the risk of chance findings. Nevertheless, although our sample size will exceed the level of a pilot-study, especially for the prediction measures that we will identify we will need new samples to replicate our findings.

Finally, next to our a priori power analyses, we will perform post hoc power analyses of our outcomes once the data have been analysed.

\section{Descriptive data}

We will provide descriptive statistics and compare groups using $\chi^{2}$ tests and independent samples $t$ tests where applicable.

\section{First and second hypotheses}

For the first hypotheses we will compare the remitted recurrent MDD-group with the control-group using (multiple) general linear models or linear mixed models (eg, complex repeated measures/covariance structure, nested data, missing data), where applicable. ${ }^{189}$ We will present results uncorrected and corrected for confounders (factors differing between groups with $\mathrm{p}<0.1$ ) and/ or covariates of interest, using propensity scores where applicable. ${ }^{190}$ Independent variables will be group (recurrent MDD vs control), potential covariates, their interaction(s) and confounders; the selected outcome(s) for a given specific hypothesis will be dependent variable 
(s). If interaction effects do not contribute to the model, we will remove them to obtain the most parsimonious models. For the second hypotheses we will use comparable models, except that we will omit the control-group (and consequently the group-variable and interactions) from the models, and focus on effects of clinical variables of interest in the remitted recurrent MDD-group.

\section{Third and fourth hypotheses}

For the prediction analyses, we will use cox-regression models to investigate prospective association between baseline measures and time until first recurrence. Using time until first recurrence as primary outcome measure will provide additional modelable variance in the data since such contrasts not only incorporate $50 \%$ recurrence, but also fast versus slow recurrence that may be highly relevant from a clinical perspective. Furthermore, in first instance we are planning to only use the time invariant baseline predictors. However, in a later stage, we will incorporate the variables that we measure over time, for example, the HDRS or rumination questionnaires, to see how changes in these parameters over time are associated with future recurrence (eg, mediation) and/or time until recurrence.

Next, we will model significant univariate associations in multiple regression models, with correction for other confounders and covariates of interest related to recurrence (eg, number of previous episodes, residual symptoms, 'daily hassles' and coping style). Moreover, we will analyse secondary outcomes (cumulative number, length and severity of MDD-episodes and course of depressive (residual) symptoms) using (multivariate) general linear models or linear mixed models, where applicable. For the fourth hypotheses, we will investigate change during recurrence using repeated measures general linear models or linear mixed models where applicable.

\section{Additional analyses}

To exploit the multimodal and multidimensional character of our data, we plan to apply advanced statistical methods to identify relevant multivariate patterns, including machine learning, factor and network analyses. ${ }^{191-193}$

\section{ETHICS AND DISSEMINATION Ethical considerations \\ Regulation statement}

We will conduct this study according to Declaration of Helsinki principles (Seoul, October 2008) and the Medical Research Involving Human Subjects Act (WMO). The study is approved by the accredited Medical Ethical Committee (METC) of the Academic Medical Centre (AMC), teaching hospital of the University of Amsterdam. We will obtain written informed consent beforehand from all participants, after careful and extensive written and oral information. If desired, we will give participants up to 2 weeks to consider their decision. Investigators will receive good clinical practice training, in agreement with the AMC research code.

\section{Handling of data and documents}

We will encode data and keep this data and blood samples for at least 15 years. Only researchers directly involved in the study will have access to encoded data, the key will be with the researcher only. We will label blood samples with anonymised patient numbers.

\section{Benefits and risk assessment}

There is no immediate advantage of participation for participants, there are no interventions scheduled in this study. MRI is non-invasive, so hardly any risks are associated with this study. Therefore, the METC determined that no liability insurance is required. We will inform participants and the reviewing accredited METC if anything occurs, on the basis of which it appears that disadvantages of participation may be significantly greater than was foreseen.

Because we recruit unmedicated participants with moderate to high recurrence risk, it may be questioned whether follow-up of these participants is ethically justified. However, we will not actively propose tapering or discontinuation of antidepressant therapy. Instead we will only include participants who decided to stop antidepressants beforehand. In case we detect suicidality during follow-up, we have a protocol available including a consulting psychiatrist for emergency situations and referral the most appropriate emergency service. We therefore consider this study ethically justifiable.

In addition, advantages of participation and follow-up will be that MDD-recurrence will be detected early so prompt psychiatric treatment can be offered. In naturalistic care there might be substantial patient and institutional delays before recurrence is detected and treatment can be started. ${ }^{194}$

\section{Compensation}

Participants will receive $€ 75$,- for their participation, besides compensation for travel expenses. For completion of a follow-up scan we will pay $€ 50,-$.

\section{Teaching}

This study will provide training of PhD-students, and will involve educational internships of medicine, psychology and neuroscience bachelor-students and master-students of the Universities of Amsterdam, Nijmegen and Groningen and VU-university.

\section{Dissemination}

Public disclosure and publication policy

We will submit study-results for publication in peer reviewed journals and presentation at (inter)national meetings, taking into account relevant reporting guidelines (eg, Committee on Publication Ethics (COPE), STrengthening the Reporting of OBservational studies 
in Epidemiology (STROBE)). ${ }^{195-197}$ We will regularly notify participants of publication. Curated technical appendices, statistical code, and anonymised data will become freely available from the corresponding authors on request. ${ }^{198}$

\section{DISCUSSION}

\section{Summary}

In summary, the current multimodal DELTAneuroimaging study will investigate recurrent MDD vulnerability by comparing remitted unmedicated recurrent MDD-participants with carefully matched controls without personal/first degree familial psychiatric history. Biopsychosocial assessments integrate four distinct levels of perspective: symptomatology, affective neuropsychology, brain circuits and endocrinology/metabolism. Subsequently, the cohort of recurrent MDD-participants will be followed-up to test to what extent baseline measurements predict, and/or change during prospective recurrence. This will help to disentangle the pathophysiology behind MDD-recurrence, and thereby provide (1) (bio) markers identifying high-risk patients needing additional preventive treatment, and (2) novel targets to improve the treatments preventing against recurrences. Given MDD's highly recurrent nature, this knowledge has the potential to substantially reduce MDD's disease burden.

\section{Limitations and strengths \\ Limitations}

Several limitations of the current study should be noted beforehand. First, the extensive assessment procedure needed to measure all variables of interest and confounders will potentially lead to inclusion of participants that are intrinsically aware of the necessity to perform clinical research and readily willing to cooperate. Nevertheless, this selection bias is inherent to translational neuroscientific research, and the relatively large number of participants that will be included will increase external validity. Moreover, testing the integrated hypotheses of the current study is only possible by combining the different assessments.

Second, to overcome potential confounding effects of antidepressants and other psychotropic medication, only participants who currently do not use these drugs will be included. This may lead to selection of particular patient subgroups that (1) experienced little benefit from previous medication trials, (2) are hesitant to use these medications because of adverse effects or for principle reasons, (3) experience other barriers to care (eg, financial) or (4) have an intrinsically lower vulnerability to have severe recurrences. In addition, it may slow down inclusion. However, this is the only way to study the hypotheses at hand while eliminating confounding effects of medication use. Furthermore, the participants included in the current study may be a clinically relevant representation of patients that do not want to take antidepressant drugs, for whom knowledge of underlying vulnerability and measures to determine this vulnerability might be of help to develop novel alternative treatments to prevent recurrence risk.

Third, for practical reasons family history will be determined by heteroanamnesis. This may lead to recall or other biases. However, both under and overrepresentation can be expected, so we expect this will not result in systematic biases.

Fourth, DSM-IV diagnostic criteria will be used for current study's diagnoses, while the DSM- 5 has already been introduced. Since the classification of depressive episodes (ie, recurrences) have not changed in DSM-5, this and our specific inclusion and exclusion criteria will not lead to difficulties in translating the results when DSM- 5 will be used.

Fifth, the current study's assessments will not include measures of HPA-axis feedback (e.g. dexamethasone suppression (/corticotropin-releasing hormone-challenge) test). ${ }^{199}$ This was not included to prevent overburdening of participants. While consequently the current study will not be able to directly assess HPA-axis feedback, the study's seven salivary HPA-axis measures without pharmacological challenge during the baseline assessments will provide an adequate indication of HPA-axis activity under natural circumstances, including stress by mood induction. ${ }^{120} 172$

Sixth, the current study's MRI-measures will be made using $3 \mathrm{~T}$ field strength, while higher field strengths are also available. Although obviously higher field strengths increase signal to noise ratio, they may also have several disadvantages. ${ }^{200}$ Higher costs and specific absorption rates, together with increased risk for artefacts due to for example, inhomogeneous transmit fields, more extensive contraindications and peripheral nerve stimulation limit high field strength applicability. These disadvantages apply to clinical studies like the present one, but even more to the clinical setting. ${ }^{200}$ Therefore, $3 \mathrm{~T}$ findings may be more readily clinically translated than findings at higher field strengths, and could therefore be more relevant from the clinical perspective. ${ }^{200}$

Seventh, while the combined cross-sectional patientcontrol and prospective follow-up design of the current study has great advantages, it brings along a balance between two contrasts. First, the recurrent MDDvulnerability contrast in the comparison between highly vulnerable patients and matched resilient controls; and second the within patient-group contrast in time until recurrence of fast recurrence during follow-up versus no or late recurrence. Strongly increasing the first contrast by including only extremely high recurrence risk patients entails the risk of decreasing the second contrast because all patients will experience fast recurrence. The other way around, by including too many patients with a low recurrence risk, the first contrast may be disadvantaged because the traits will not be outspoken enough to be detected. Therefore, also based on our previous research, we opted to increase to first contrast by including relatively resilient controls, together with 
patients that have proven vulnerability for recurrent MDD (ie, $\leq 2$ previous MDD episodes). However, we did not express any additional vulnerability criteria, for example, time since last episode or higher number of previous episodes, in order to (1) include recurrent MDD-patients that form a naturalistic sample that is representative regarding vulnerability and (2) not to decrease the second contrast in time until recurrence.

Of note, we will not include single episode MDD-participants. While this would enable comparisons against a relatively low recurrence risk group, instead of controls, this was deemed to be logistically even more difficult to achieve. Regarding the second contrast in time until recurrence, based on our previous research and our inclusion procedure, we expect a large spread in the number of previous episodes (eg, from 2 up to 60 ) and time since last episode (eg, from 8 weeks up to $>10$ years), which both imply modelable variance/contrast in prospective recurrence risk. ${ }^{201}$ With an expected 'optimal' distribution of $50 \%$ recurrence-rate during follow-up, we think that our group would be the most interesting and feasible group to study when looking for factors that can predict imminent recurrence, in order to (1) select participants that may benefit from preventive treatment, and (2) identify pathophysiological mechanisms that can be targeted in these participants to prevent recurrence risk.

Finally, the current study does not include (randomised) interventions. Therefore, it will not be possible to say whether observed effects are causal in nature. Nevertheless, the current study's prospective, repeated measures design can optimally select targets for future randomised clinical trials to test the causal nature of observed effects.

\section{Strengths}

The current study also has several distinct strengths. Owing to its strict and specific inclusion-criteria, matching-procedure and recruitment-procedure, the contrast for MDD-vulnerability will be maximal, without distortion due to important confounders: MDD-residual symptoms and medication. In addition, the unique integration of a wide range of measures in a prospective repeated measures design will allow disentangling of recurrent MDD state-factors and trait-factors.

Furthermore, the study will be performed by an experienced international multicentre research group, combining expertise from all measured perspectives. Additionally, the Netherlands' relative limited geographic size and high level of social organisation make it well suited for long-term follow-up research.

Next, ESM-results could set the stage for innovative cost-effective e-health interventions. Moreover, the focus on lifestyle factors (physical activity/diet) and their biological effects could provide more insight into recurrent MDD-patients' increased risk to develop cardiovascular disease, ${ }^{9}$ as already acknowledged in the introduction. By combining these lifestyle (biological) assessments with investigation of (the neurobiology of) motivation, the present study could lead to development of interventions that help to motivate recurrent MDD-patients to improve their lifestyle. This not only has the potential to prevent recurrence, but also the highly comorbid cardiovascular risk. ${ }^{202}$

\section{Conclusion}

By integrating the symptom level, affective neuropsychology, brain circuits and endocrinology/metabolism, using a prospective repeated measures design in remitted MDD-participants, the present DELTA-neuroimaging study will provide more insight in recurrent MDD-vulnerability. Increased insight will lead to novel targets for (I) improved preventive therapy, and/or (II) (bio) markers to monitor and/or predict recurrence risk. Consequently, ultimately, it holds potential to alleviate MDD's highly recurrent course and reduce its currently overwhelming global disease burden.

\section{Author affiliations}

${ }^{1}$ Department of Psychiatry, Academic Medical Center, University of Amsterdam, The Netherlands

${ }^{2}$ University of Groningen, Neuroimaging Center, University Medical Center Groningen, The Netherlands

${ }^{3}$ Program for Mood and Anxiety Disorders, Department of Psychiatry, University of Groningen, University Medical Center Groningen, The Netherlands

${ }^{4}$ Laboratory Genetic Metabolic Disease, Academic Medical Center, University of Amsterdam, The Netherlands

${ }^{5}$ University of Groningen, Interdisciplinary Center Psychopathology and Emotion regulation (ICPE), University Medical Center Groningen, The Netherlands

${ }^{6}$ Laboratory of General Clinical Chemistry, Academic Medical Center, University of Amsterdam, The Netherlands

${ }^{7}$ Department of Experimental Clinical and Health Psychology, Ghent University, Belgium

${ }^{8}$ Department of Clinical Psychology, University of Groningen, Groningen, The Netherlands

${ }^{9}$ Department of Clinical and Health Psychology, Utrecht University, Utrecht, The Netherlands

${ }^{10}$ Department of Psychiatry, University of Oxford, Warneford Hospital, Oxford, UK

${ }^{11}$ Department of Psychiatry, Radboud University Medical Center, Nijmegen, The Netherlands

${ }^{12}$ Donders Institute for Brain, Cognition and Behavior, Radboud University Nijmegen, Nijmegen, The Netherlands

Contributors RJTM and HGR designed the study. RJTM and HGR drafted the protocol and the manuscript. All authors contributed to development and implementation of the study protocol. MWJK provided statistical advice. RJTM and CAF conduct all participant-related study-procedures. All authors contributed to editing the manuscript and read and approved the final manuscript.

Funding This study is supported by unrestricted personal grants from the AMC to RJTM (AMC PhD Scholarship) and CAF (AMC MD-PhD Scholarship), and a dedicated grant from the Dutch Brain Foundation (Hersenstichting Nederland: 2009(2)-72). HGR is supported by a NWO/ZonMW VENI-Grant \#016.126.059.

Competing interests None declared.

Patient consent Obtained.

Ethics approval The local ethics committee of the Academic Medical Center of the University of Amsterdam approved this study (AMC-METC-Nr.:11/050). 
Provenance and peer review Not commissioned; externally peer reviewed.

Data sharing statement We will submit study-results for publication in peer reviewed journals and presentation at (inter)national meetings, taking into account relevant reporting guidelines (eg, COPE, STROBE). We will regularly notify participants of publication. Curated technical appendices, statistical code, and anonymised data will become freely available from the corresponding authors on request.

Open Access This is an Open Access article distributed in accordance with the Creative Commons Attribution Non Commercial (CC BY-NC 4.0) license, which permits others to distribute, remix, adapt, build upon this work noncommercially, and license their derivative works on different terms, provided the original work is properly cited and the use is non-commercial. See: http:// creativecommons.org/licenses/by-nc/4.0/

\section{REFERENCES}

1. Andrade L, Caraveo-Anduaga JJ, Berglund $\mathrm{P}$, et al. The epidemiology of major depressive episodes: results from the International Consortium of Psychiatric Epidemiology (ICPE) Surveys. Int J Methods Psychiatr Res 2003;12:3-21.

2. Greden JF. The burden of recurrent depression: causes, consequences, and future prospects. J Clin psychiatry 2001;62 (Suppl 22):5-9.

3. Vos T, Flaxman AD, Naghavi M, et al. Years lived with disability (YLDs) for 1160 sequelae of 289 diseases and injuries 1990-2010: a systematic analysis for the Global Burden of Disease Study 2010. Lancet 2012;380:2163-96.

4. Bromet $\mathrm{E}$, Andrade LH, Hwang I, et al. Cross-national epidemiology of DSM-IV major depressive episode. BMC Med 2011:9:90.

5. Mathers CD, Loncar D. Projections of global mortality and burden of disease from 2002 to 2030. PLoS Med 2006;3:e442.

6. Cuijpers P, Smit F, Oostenbrink J, et al. Economic costs of minor depression: a population-based study. Acta Psychiatr Scand 2007;115:229-36.

7. Sobocki $\mathrm{P}$, Ekman $\mathrm{M}$, Agren $\mathrm{H}$, et al. The mission is remission: health economic consequences of achieving full remission with antidepressant treatment for depression. Int J Clin Pract 2006;60:791-8.

8. Sobocki P, Jonsson B, Angst J, et al. Cost of depression in Europe. $J$ Ment Health Policy Econ 2006;9:87-98.

9. Assies J, Mocking RJ, Lok A, et al. Effects of oxidative stress on fatty acid- and one-carbon-metabolism in psychiatric and cardiovascular disease comorbidity. Acta Psychiatr Scand 2014;130:163-80.

10. Kraepelin E, Barclay RM, Robertson GM. Manic-depressive insanity and paranoia. Edinburgh: Livingstone, 1921

11. Angst J, Baastrup $\mathrm{P}$, Grof $\mathrm{P}$, et al. The course of monopolar depression and bipolar psychoses. Psychiatr Neurol Neurochir 1973;76:489-500.

12. Geddes JR, Carney SM, Davies C, et al. Relapse prevention with antidepressant drug treatment in depressive disorders: a systematic review. Lancet 2003;361:653-61.

13. Kupfer DJ. Long-term treatment of depression. J Clin Psychiatry 1991;52(Suppl):28-34.

14. Prien RF, Carpenter LL, Kupfer DJ. The definition and operational criteria for treatment outcome of major depressive disorder. A review of the current research literature. Arch Gen Psychiatry 1991;48:796-800.

15. Monroe SM, Harkness KL. Recurrence in major depression: a conceptual analysis. Psychol Rev 2011;118:655-74.

16. Steinert C, Hofmann M, Kruse J, et al. The prospective long-term course of adult depression in general practice and the community. A systematic literature review. J Affect Disord 2014;152-154:65-75.

17. Eaton WW, Shao $\mathrm{H}$, Nestadt $\mathrm{G}$, et al. Population-based study of first onset and chronicity in major depressive disorder. Arch Gen Psychiatry 2008:65:513-20.

18. Hardeveld F, Spijker J, De Graaf R, et al. Recurrence of major depressive disorder and its predictors in the general population: results from the Netherlands Mental Health Survey and Incidence Study (NEMESIS). Psychol Med 2013;43:39-48.

19. Hardeveld F, Spijker J, De Graaf R, et al. Prevalence and predictors of recurrence of major depressive disorder in the adult population. Acta Psychiatr Scand 2010;122:184-91.

20. Mueller TI, Leon AC, Keller MB, et al. Recurrence after recovery from major depressive disorder during 15 years of observational follow-up. Am J Psychiatry 1999;156:1000-6.
21. Bhagwagar Z, Cowen PJ. 'It's not over when it's over': persistent neurobiological abnormalities in recovered depressed patients. Psychol Med 2008;38:307-13.

22. Bockting $\mathrm{CL}$, Smid $\mathrm{NH}$, Koeter MW, et al. Enduring effects of Preventive Cognitive Therapy in adults remitted from recurrent depression: A 10 year follow-up of a randomized controlled trial. $J$ Affect Disord 2015;185:188-94.

23. Judd LL, Akiskal HS, Zeller PJ, et al. Psychosocial disability during the long-term course of unipolar major depressive disorder. Arch Gen Psychiatry 2000;57:375-80.

24. Kaymaz N, van Os J, Loonen AJ, et al. Evidence that patients with single versus recurrent depressive episodes are differentially sensitive to treatment discontinuation: a meta-analysis of placebocontrolled randomized trials. J Clin Psychiatr 2008;69:1423-36.

25. Bockting CL, ten Doesschate MC, Spijker J, et al. Continuation and maintenance use of antidepressants in recurrent depression. Psychother Psychosom 2008;77:17-26.

26. ten Doesschate MC, Bockting CL, Koeter MW, et al. Predictors of nonadherence to continuation and maintenance antidepressant medication in patients with remitted recurrent depression. J Clin Psychiatry 2009;70:63-9.

27. ten Doesschate $\mathrm{MC}$, Bockting $\mathrm{CL}$, Schene $\mathrm{AH}$. Adherence to continuation and maintenance antidepressant use in recurrent depression. J AffectDisord 2009;115:167-70.

28. Vittengl JR, Clark LA, Dunn TW, et al. Reducing relapse and recurrence in unipolar depression: a comparative meta-analysis of cognitive-behavioral therapy's effects. J Consult Clin Psychol 2007;75:475-88.

29. Piet J, Hougaard E. The effect of mindfulness-based cognitive therapy for prevention of relapse in recurrent major depressive disorder: a systematic review and meta-analysis. Clin Psychol Rev 2011;31:1032-40.

30. Bockting $\mathrm{CL}$, Schene $\mathrm{AH}$, Spinhoven $\mathrm{P}$, et al. Preventing relapse/ recurrence in recurrent depression with cognitive therapy: a randomized controlled trial. J Consult Clin Psychol 2005;73: 647-57.

31. Bockting CL, Spinhoven P, Wouters LF, et al. Long-term effects of preventive cognitive therapy in recurrent depression: a 5.5-year follow-up study. J Clin Psychiatry 2009;70:1621-8.

32. Teasdale JD, Scott J, Moore RG, et al. How does cognitive therapy prevent relapse in residual depression? Evidence from a controlled trial. J Consult Clin Psychol 2001;69:347-57.

33. Teasdale JD, Segal ZV, Williams JM, et al. Prevention of relapse/ recurrence in major depression by mindfulness-based cognitive therapy. J Consult Clin Psychol 2000;68:615-23.

34. Huijbers MJ, Spijker J, Donders AR, et al. Preventing relapse in recurrent depression using mindfulness-based cognitive therapy, antidepressant medication or the combination: trial design and protocol of the MOMENT study. BMC Psychiatry 2012;12:125

35. Kuyken W, Byford S, Taylor RS, et al. Mindfulness-based cognitive therapy to prevent relapse in recurrent depression. J Consult Clin Psychol 2008;76:966-78.

36. Biesheuvel-Leliefeld KE, Kok GD, Bockting CL, et al. Effectiveness of psychological interventions in preventing recurrence of depressive disorder: Meta-analysis and meta-regression. J Affect Disord 2014:174C:400-10.

37. Steinert C, Hofmann M, Kruse J, et al. Relapse rates after psychotherapy for depression-stable long-term effects? A meta-analysis. J Affect Disord 2014;168:107-18.

38. Bockting CL, Mocking RJ, Lok A, et al. Therapygenetics: the 5HTTLPR as a biomarker for response to psychological therapy? MolPsychiatry 2013;18:744-5.

39. Kessing LV, Hansen MG, Andersen PK, et al. The predictive effect of episodes on the risk of recurrence in depressive and bipolar disorders-a life-long perspective. Acta Psychiatr Scand 2004; 109:339-44.

40. Pettit JW, Lewinsohn PM, Joiner TE Jr. Propagation of major depressive disorder: relationship between first episode symptoms and recurrence. Psychiatry Res 2006;141:271-8.

41. Pettit JW, Hartley C, Lewinsohn PM, et al. Is liability to recurrent major depressive disorder present before first episode onset in adolescence or acquired after the initial episode? J Abnorm Psychol 2013;122:353-8.

42. Bulloch $\mathrm{A}$, Williams $\mathrm{J}$, Lavorato $\mathrm{D}$, et al. Recurrence of major depressive episodes is strongly dependent on the number of previous episodes. Depress Anxiety 2014;31:72-6.

43. Crona L, Bradvik L. Long-term course of severe depression: late remission and recurrence May be found in a follow-up after 38-53 years. Ment IIIn 2012;4:e17.

44. Monroe SM, Harkness KL. Is depression a chronic mental illness? Psychol Med 2012;42:899-902. 
45. Colman I, Naicker $\mathrm{K}$, Zeng $\mathrm{Y}$, et al. Predictors of long-term prognosis of depression. CMAJ 2011;183:1969-76.

46. Merikangas $\mathrm{KR}$, Zhang $\mathrm{H}$, Avenevoli $\mathrm{S}$, et al. Longitudinal trajectories of depression and anxiety in a prospective community study: the Zurich Cohort Study. Arch Gen Psychiatry 2003;60:993-1000.

47. Bockting CL, Spinhoven P, Koeter MW, et al. Prediction of recurrence in recurrent depression and the influence of consecutive episodes on vulnerability for depression: a 2-year prospective study. J Clin Psychiatry 2006;67:747-55.

48. ten Doesschate MC, Bockting CL, Koeter MW, et al. Prediction of recurrence in recurrent depression: a 5.5-year prospective study. $J$ Clin Psychiatry 2010;71:984-91.

49. Judd LL, Paulus, MJ, Schettler, PJ, et al. Does incomplete recovery from first lifetime major depressive episode herald a chronic course of illness? Am J Psychiatry 2000;157:1501-4.

50. Scher CD, Ingram RE, Segal ZV. Cognitive reactivity and vulnerability: empirical evaluation of construct activation and cognitive diatheses in unipolar depression. Clin Psychol Rev 2005;25:487-510.

51. Kendler KS, Thornton LM, Gardner CO. Stressful life events and previous episodes in the etiology of major depression in women: an evaluation of the "kindling" hypothesis. Am J Psychiatry 2000;157:1243-51.

52. Segal ZV, Williams JM, Teasdale JD, et al. A cognitive science perspective on kindling and episode sensitization in recurrent affective disorder. Psychol Med 1996;26:371-80.

53. Schumann G, Binder EB, Holte A, et al. Stratified medicine for mental disorders. Eur Neuropsychopharmacol 2014;24:5-50.

54. Insel TR. The NIMH Research Domain Criteria (RDoC) Project: precision medicine for psychiatry. Am J Psychiatry 2014;171:395-7.

55. Teasdale JD. Cognitive vulnerability to persistent depression. Cogn Emotion 1988;2:247-74.

56. Segal ZV, Kennedy S, Gemar M, et al. Cognitive reactivity to sad mood provocation and the prediction of depressive relapse. Arch Gen Psychiatry 2006;63:749-55.

57. van Rijsbergen GD, Bockting $\mathrm{CL}$, Burger $\mathrm{H}$, et al. Mood reactivity rather than cognitive reactivity is predictive of depressive relapse: a randomized study with 5.5-year follow-up. J Consult Clin Psychol 2013;81:508-17.

58. Michalak J, Hölz A, Teismann T. Rumination as a predictor of relapse in mindfulness-based cognitive therapy for depression. Psychol Psychother 2011;84:230-6.

59. Whitton AE, Treadway MT, Pizzagalli DA. Reward processing dysfunction in major depression, bipolar disorder and schizophrenia. Curr Opin Psychiatry 2015;28:7-12.

60. Eshel N, Roiser JP. Reward and punishment processing in depression. Biol Psychiatry 2010;68:118-24.

61. Pizzagalli DA. Depression, stress, and anhedonia: toward a synthesis and integrated model. Annu Rev Clin Psychol 2014:10:393-423

62. Treadway MT, Zald DH. Reconsidering anhedonia in depression: lessons from translational neuroscience. Neurosci Biobehav Rev 2011:35:537-55.

63. Fredrickson BL. The role of positive emotions in positive psychology. The broaden-and-build theory of positive emotions. Am Psychol 2001;56:218-26.

64. Mathews A, MacLeod C. Cognitive approaches to emotion and emotional disorders. Annu Rev Psychol 1994;45:25-50.

65. De Raedt R, Koster EH. Understanding vulnerability for depression from a cognitive neuroscience perspective: a reappraisal of attentional factors and a new conceptual framework. Cogn Affect Behav Neurosci 2010;10:50-70.

66. Leyman L, De Raedt R, Schacht R, et al. Attentional biases for angry faces in unipolar depression. Psychol Med 2007;37:393-402.

67. Goeleven E, De Raedt R, Baert S, et al. Deficient inhibition of emotional information in depression. J Affect Disord 2006;93:149-57.

68. Joormann J, Gotlib IH. Selective attention to emotional faces following recovery from depression. J Abnorm Psychol 2007;116:80-5.

69. Harmer CJ, Goodwin GM, Cowen PJ. Why do antidepressants take so long to work? A cognitive neuropsychological model of antidepressant drug action. Br J Psychiatry 2009;195:102-8.

70. DeRubeis RJ, Siegle GJ, Hollon SD. Cognitive therapy versus medication for depression: treatment outcomes and neural mechanisms. Nat Rev Neurosci 2008;9:788-96.

71. Harmer CJ, O'Sullivan U, Favaron E, et al. Effect of acute antidepressant administration on negative affective bias in depressed patients. Am J Psychiatry 2009;166:1178-84.

72. Pizzagalli DA, Peccoralo LA, Davidson RJ, et al. Resting anterior cingulate activity and abnormal responses to errors in subjects with elevated depressive symptoms: a 128-channel EEG study. Hum Brain Mapp 2006;27:185-201.
73. Nolen-Hoeksema S. The role of rumination in depressive disorders and mixed anxiety/depressive symptoms. J Abnorm Psychol 2000;109:504-11.

74. Ingram RE. Origins of cognitive vulnerability to depression. Cognit Ther Res 2003;27:77-88.

75. Nejad AB, Fossati P, Lemogne C. Self-referential processing, rumination, and cortical midline structures in major depression. Front Hum Neurosci 2013;7:666.

76. Lau MA, Segal ZV, Williams JM. Teasdale's differential activation hypothesis: implications for mechanisms of depressive relapse and suicidal behaviour. Behav Res Ther 2004;42:1001-17.

77. Bouhuys AL, Geerts E, Gordijn MC. Depressed patients perceptions of facial emotions in depressed and remitted states are associated with relapse: a longitudinal study. J Nerv Ment Dis 1999;187:595-602.

78. Nandrino JL, Dodin V, Martin P, et al. Emotional information processing in first and recurrent major depressive episodes. J Psychiatr Res 2004;38:475-84.

79. Leppanen JM. Emotional information processing in mood disorders: a review of behavioral and neuroimaging findings. Curr Opin Psychiatry 2006;19:34-9.

80. Elgersma HJ, Glashouwer KA, Bockting CL, et al. Hidden scars in depression? Implicit and explicit self-associations following recurrent depressive episodes. J Abnorm Psychol 2013;122:951-60.

81. Phillips ML, Drevets WC, Rauch SL, et al. Neurobiology of emotion perception II: implications for major psychiatric disorders. Biol Psychiatry 2003;54:515-28.

82. Phillips ML, Ladouceur CD, Drevets WC. A neural model of voluntary and automatic emotion regulation: implications for understanding the pathophysiology and neurodevelopment of bipolar disorder. Mol Psychiatry 2008;13:829, 33-57.

83. Mayberg HS. Modulating dysfunctional limbic-cortical circuits in depression: towards development of brain-based algorithms for diagnosis and optimised treatment. Br Med Bull 2003;65: 193-207.

84. Pizzagalli DA. Frontocingulate dysfunction in depression: toward biomarkers of treatment response. Neuropsychopharmacology 2011;36:183-206.

85. Surguladze S, Brammer MJ, Keedwell P, et al. A differential pattern of neural response toward sad versus happy facial expressions in major depressive disorder. Biol Psychiatry 2005;57:201-9.

86. Ruhé HG, Booij J, Veltman DJ, et al. Successful pharmacologic treatment of major depressive disorder attenuates amygdala activation to negative facial expressions: a functional magnetic resonance imaging study. J Clin Psychiatry 2012;73:451-9.

87. Groenewold NA, Opmeer EM, de Jonge P, et al. Emotional valence modulates brain functional abnormalities in depression: evidence from a meta-analysis of fMRI studies. Neurosci Biobehav Rev 2013;37:152-63.

88. Rive MM, van Rooijen G, Veltman DJ, et al. Neural correlates of dysfunctional emotion regulation in major depressive disorder. A systematic review of neuroimaging studies. Neurosci Biobehav Rev 2013;37(10 Pt 2):2529-53.

89. Frodl TS, Koutsouleris N, Bottlender R, et al. Depression-related variation in brain morphology over 3 years: effects of stress? Arch Gen Psychiatry 2008;65:1156-65.

90. Marchetti I, Koster EH, Sonuga-Barke EJ, et al. The default mode network and recurrent depression: a neurobiological model of cognitive risk factors. Neuropsychol Rev 2012;22:229-51.

91. Chai XJ, Castanon AN, Ongur D, et al. Anticorrelations in resting state networks without global signal regression. Neurolmage 2012;59:1420-8.

92. Fox MD, Raichle ME. Spontaneous fluctuations in brain activity observed with functional magnetic resonance imaging. Nat Rev Neurosci 2007;8:700-11.

93. Fox MD, Snyder AZ, Vincent JL, et al. The human brain is intrinsically organized into dynamic, anticorrelated functional networks. Proc Natl Acad Sci USA 2005;102:9673-8.

94. Fransson P. Spontaneous low-frequency BOLD signal fluctuations: An fMRI investigation of the resting-state default mode of brain function hypothesis. Hum Brain Mapp 2005;26:15-29.

95. Anticevic A, Cole MW, Murray JD, et al. The role of default network deactivation in cognition and disease. Trends Cogn Sci (Regul Ed) 2012;16:584-92.

96. Kaiser RH, Andrews-Hanna JR, Wager TD, et al. Large-Scale Network Dysfunction in Major Depressive Disorder: A Meta-analysis of Resting-State Functional Connectivity. JAMA Psychiatry 2015;72:603-11.

97. Sheline $\mathrm{YI}$, Price JL, Yan Z, et al. Resting-state functional MRI in depression unmasks increased connectivity between networks via the dorsal nexus. Proc Natl Acad Sci USA 2010;107:11020-5. 
98. Greicius MD, Flores BH, Menon V, et al. Resting-state functional connectivity in major depression: abnormally increased contributions from subgenual cingulate cortex and thalamus. Biol Psychiatry 2007;62:429-37.

99. Zhou Y, Yu C, Zheng H, et al. Increased neural resources recruitment in the intrinsic organization in major depression. $J$ Affect Disord 2010;121:220-30.

100. Grimm S, Boesiger P, Beck J, et al. Altered negative BOLD responses in the default-mode network during emotion processing in depressed subjects. Neuropsychopharmacology 2009;34:932-43.

101. Raichle ME, Gusnard DA. Intrinsic brain activity sets the stage for expression of motivated behavior. J Comp Neurol 2005;493:167-76.

102. Belleau EL, Taubitz LE, Larson CL. Imbalance of default mode and regulatory networks during externally focused processing in depression. Soc Cogn Affect Neurosci 2015;10:744-51.

103. Farb NA, Anderson AK, Bloch RT, et al. Mood-linked responses in medial prefrontal cortex predict relapse in patients with recurrent unipolar depression. Biol Psychiatry 2011;70:366-72.

104. Zhu X, Wang X, Xiao J, et al. Evidence of a dissociation pattern in resting-state default mode network connectivity in first-episode, treatment-naive major depression patients. Biol Psychiatry 2012;71:611-7.

105. Berman MG, Peltier S, Nee DE, et al. Depression, rumination and the default network. Soc Cogn Affect Neurosci 2011;6:548-55.

106. Hamilton JP, Furman DJ, Chang C, et al. Default-mode and task-positive network activity in major depressive disorder: implications for adaptive and maladaptive rumination. Biol Psychiatry 2011;70:327-33.

107. Pechtel P, Dutra SJ, Goetz EL, et al. Blunted reward responsiveness in remitted depression. J Psychiatr Res 2013;47:1864-9.

108. Kumar $\mathrm{P}$, Waiter $\mathrm{G}$, Ahearn $\mathrm{T}$, et al. Abnormal temporal difference reward-learning signals in major depression. Brain 2008;131(Pt 8):2084-93.

109. Morgan JK, Olino TM, McMakin DL, et al. Neural response to reward as a predictor of increases in depressive symptoms in adolescence. Neurobiol Dis 2013;52:66-74.

110. Hall GB, Milne AM, Macqueen GM. An fMRI study of reward circuitry in patients with minimal or extensive history of major depression. Eur Arch Psychiatry Clin Neurosci 2014;264:187-98.

111. Ramel W, Goldin PR, Eyler LT, et al. Amygdala reactivity and mood-congruent memory in individuals at risk for depressive relapse. Biol Psychiatry 2007:61:231-9.

112. Hooley JM, Gruber SA, Parker HA, et al. Cortico-limbic response to personally challenging emotional stimuli after complete recovery from depression. Psychiatry Res 2009;172:83-91.

113. Hooley JM, Gruber SA, Scott LA, et al. Activation in dorsolateral prefrontal cortex in response to maternal criticism and praise in recovered depressed and healthy control participants. Biol Psychiatry 2005;57:809-12.

114. Levesque J, Eugene F, Joanette $\mathrm{Y}$, et al. Neural circuitry underlying voluntary suppression of sadness. Biol Psychiatry 2003;53:502-10.

115. van Tol MJ, van der Wee NJ, van den Heuvel OA, et al. Regional brain volume in depression and anxiety disorders. Arch Gen Psychiatry 2010;67:1002-11.

116. Frodl T, Meisenzahl EM, Zetzsche T, et al. Hippocampal and amygdala changes in patients with major depressive disorder and healthy controls during a 1-year follow-up. J Clin Psychiatry 2004:65:492-9.

117. Kronmuller KT, Pantel J, Kohler S, et al. Hippocampal volume and 2-year outcome in depression. Br J Psychiatry 2008;192:472-3.

118. Frodl T, Jager M, Born C et al. Anterior cingulate cortex does not differ between patients with major depression and healthy controls, but relatively large anterior cingulate cortex predicts a good clinical course. Psychiatry Res 2008;163:76-83.

119. Stetler C, Miller GE. Depression and hypothalamic-pituitary-adrenal activation: a quantitative summary of four decades of research. Psychosom Med 2011;73:114-26.

120. Lok A, Mocking RJ, Ruhé HG, et al. Longitudinal hypothalamicpituitary-adrenal axis trait and state effects in recurrent depression. Psychoneuroendocrinology 2012;37:892-902.

121. Assies J, Visser I, Nicolson NA, et al. Elevated salivary dehydroepiandrosterone-sulfate but normal cortisol levels in medicated depressed patients: preliminary findings. Psychiatry Res 2004:128:117-22

122. Appelhof BC, Huyser J, Verweij M, et al. Glucocorticoids and relapse of major depression (dexamethasone/ corticotropin-releasing hormone test in relation to relapse of major depression). BiolPsychiatry 2006;59:696-701.

123. Aubry JM, Gervasoni N, Osiek C, et al. The DEX/CRH neuroendocrine test and the prediction of depressive relapse in remitted depressed outpatients. J Psychiatr Res 2007;41:290-4.
124. Bockting CL, Lok A, Visser I, et al. Lower cortisol levels predict recurrence in remitted patients with recurrent depression: a 5.5 year prospective study. Psychiatry Res 2012;200:281-7.

125. Hardeveld F, Spijker J, Vreeburg SA, et al. Increased cortisol awakening response was associated with time to recurrence of major depressive disorder. Psychoneuroendocrinology 2014;50:62-71.

126. MacQueen GM, Campbell S, McEwen BS, et al. Course of illness, hippocampal function, and hippocampal volume in major depression. Proc Natl Acad Sci U S A 2003;100:1387-92.

127. McEwen BS. Mood disorders and allostatic load. Biol Psychiatry 2003:54:200-7.

128. Malhi GS, Parker GB, Greenwood J. Structural and functional models of depression: from sub-types to substrates. Acta Psychiatr Scand 2005;111:94-105.

129. Urry HL, van Reekum CM, Johnstone T, et al. Amygdala and ventromedial prefrontal cortex are inversely coupled during regulation of negative affect and predict the diurnal pattern of cortisol secretion among older adults. J Neurosci 2006;26:4415-25

130. Mocking RJ, Ruhé HG, Assies J, et al. Relationship between the hypothalamic-pituitary-adrenal-axis and fatty acid metabolism in recurrent depression. Psychoneuroendocrinology 2013:38:1607-17.

131. Mocking RJT, Assies J, Bot M, et al. Biological effects of add-on eicosapentaenoic acid supplementation in diabetes mellitus and co-morbid depression: a randomized controlled trial. PLOS ONE 2012;7:e49431.

132. Martinez M, Mougan I. Fatty acid composition of human brain phospholipids during normal development. $J$ Neurochem 1998;71:2528-33.

133. Piomelli D, Astarita G, Rapaka R. A neuroscientist's guide to lipidomics. Nat Rev Neurosci 2007;8:743-54.

134. Rao JS, Ertley RN, Lee HJ, et al. n-3 polyunsaturated fatty acid deprivation in rats decreases frontal cortex BDNF via a p38 MAPK-dependent mechanism. Mol Psychiatry 2007;12:36-46.

135. Martinowich K, Lu B. Interaction between BDNF and serotonin: role in mood disorders. Neuropsychopharmacology 2008;33:73-83.

136. Bazinet RP, Laye S. Polyunsaturated fatty acids and their metabolites in brain function and disease. Nat Rev Neurosci 2014;15:771-85.

137. Assies J, Pouwer F, Lok A, et al. Plasma and erythrocyte fatty acid patterns in patients with recurrent depression: a matched case-control study. PLOS ONE 2010;5:e10635.

138. Mocking RJ, Assies J, Koeter MW, et al. Bimodal distribution of fatty acids in recurrent major depressive disorder. Biol Psychiatry 2012;71:e3-5.

139. Mocking RJ, Assies J, Lok A, et al. Statistical methodological issues in handling of fatty acid data: percentage or concentration, imputation and indices. Lipids 2012;47:541-7.

140. Mocking RJ, Lok A, Assies J, et al. Ala54Thr fatty acid-binding protein 2 (FABP2) polymorphism in recurrent depression: associations with fatty acid concentrations and waist circumference. PLOS ONE 2013;8:e82980.

141. McNamara RK. Deciphering the role of docosahexaenoic acid in brain maturation and pathology with magnetic resonance imaging. Prostaglandins Leukot Essent Fatty Acids 2013;88:33-42.

142. Ende G, Demirakca T, Tost $\mathrm{H}$. The biochemistry of dysfunctional emotions: proton MR spectroscopic findings in major depressive disorder. Prog Brain Res 2006;156:481-501.

143. Muller N, Schwarz MJ. The immune-mediated alteration of serotonin and glutamate: towards an integrated view of depression. Mol Psychiatry 2007;12:988-1000.

144. Walter M, Henning A, Grimm S, et al. The relationship between aberrant neuronal activation in the pregenual anterior cingulate, altered glutamatergic metabolism, and anhedonia in major depression. Arch Gen Psychiatry 2009;66:478-86.

145. Northoff G, Walter M, Schulte RF, et al. GABA concentrations in the human anterior cingulate cortex predict negative BOLD responses in fMRI. Nat Neurosci 2007:10:1515-7.

146. Yildiz-Yesiloglu A, Ankerst DP. Review of $1 \mathrm{H}$ magnetic resonance spectroscopy findings in major depressive disorder: a meta-analysis. Psychiatry Res 2006;147:1-25.

147. Yuksel C, Ongur D. Magnetic resonance spectroscopy studies of glutamate-related abnormalities in mood disorders. Biol Psychiatry 2010;68:785-94.

148. Hasler G, Neumeister A, van der Veen JW, et al. Normal prefrontal gamma-aminobutyric acid levels in remitted depressed subjects determined by proton magnetic resonance spectroscopy. Biol Psychiatry 2005;58:969-73.

149. Capizzano AA, Jorge RE, Acion LC, et al. In vivo proton magnetic resonance spectroscopy in patients with mood disorders: a 
technically oriented review. J Magn Reson Imaging 2007:26:1378-89.

150. First MB, Gibbon M, Spitzer RL, et al. User Guide for the Structured Clinical Interview for DSM-IV Axis-1 Disorders.

Washington DC: American Psychiatric Association, 1996.

151. Hamilton M. A rating scale for depression. J Neurol Neurosurg Psychiatr 1960;23:56-62.

152. Bockting CL, Spinhoven P, Koeter MW, et al. Differential predictors of response to preventive cognitive therapy in recurrent depression: a 2-year prospective study. Psychother Psychosom 2006;75:229-36.

153. First MB, Pincus HA. The DSM-IV Text Revision: rationale and potential impact on clinical practice. Psychiatr Serv 2002;53:288-92.

154. Posner MI, Snyder CR, Davidson BJ. Attention and the detection of signals. J Exp Psychol 1980;109:160-74.

155. Mocking RJ, Patrick Pflanz C, Pringle A, et al. Effects of short-term varenicline administration on emotional and cognitive processing in healthy, non-smoking adults: a randomized, double-blind, study. Neuropsychopharmacology 2013;38:476-84.

156. Chambers R, Lo BCY, Allen NB. The impact of intensive mindfulness training on attentional control, cognitive style, and affect. Cognit Ther Res 2008;32:303-22.

157. De Lissnyder E, Koster EH, De Raedt R. Emotional interference in working memory is related to rumination. Cognit Ther Res 2012;36:348-57.

158. Schmand B, Bakker D, Saan R, et al. [The Dutch Reading Test for Adults: a measure of premorbid intelligence level]. Tijdschr Gerontol Geriatr 1991;22:15-9.

159. Myin-Germeys I, Oorschot M, Collip D, et al. Experience sampling research in psychopathology: opening the black box of daily life. Psychol Med 2009;39:1533-47.

160. Shiffman S, Stone AA, Hufford MR. Ecological momentary assessment. Annu Rev Clin Psychol 2008:4:1-32.

161. Wichers M, Peeters F, Geschwind N, et al. Unveiling patterns of affective responses in daily life may improve outcome prediction in depression: a momentary assessment study. J Affect Disord 2010;124:191-5

162. Csikszentmihalyi M, Larson R. Validity and reliability of the Experience-Sampling Method. J Nerv Ment Dis 1987;175:526-36.

163. Crawford JR, Henry JD. The positive and negative affect schedule (PANAS): construct validity, measurement properties and normative data in a large non-clinical sample. Br J Clin Psychol 2004;43(Pt 3):245-65.

164. Schulte RF, Lange T, Beck J, et al. Improved two-dimensional J-resolved spectroscopy. NMR Biomed 2006;19:264-70.

165. Waddell KW, Avison MJ, Joers JM, et al. A practical guide to robust detection of GABA in human brain by J-difference spectroscopy at 3 T using a standard volume coil. Magn Reson Imaging 2007:25:1032-8

166. van Loon A M, Knapen T, Scholte HS, et al. GABA shapes the dynamics of bistable perception. Curr Biol 2013;23:823-7.

167. Taylor WD, Hsu E, Krishnan KR, et al. Diffusion tensor imaging: background, potential, and utility in psychiatric research. Biol Psychiatry 2004;55:201-7.

168. Vanderhasselt MA, Baeken C, Van Schuerbeek $P$, et al. How brooding minds inhibit negative material: an event-related fMRI study. Brain Cogn 2013;81:352-9.

169. Lok A, Bockting CL, Koeter MW, et al. Interaction between the MTHFR C677T polymorphism and traumatic childhood events predicts depression. Transl Psychiatry 2013;3:e288.

170. Lok A, Mocking RJ, Assies J, et al. The one-carbon-cycle and methylenetetrahydrofolate reductase (MTHFR) C677T polymorphism in recurrent major depressive disorder; influence of antidepressant use and depressive state? J Affect Disord 2014;166:115-23

171. Naviaux RK. Metabolic features of the cell danger response. Mitochondrion 2014;16:7-17.

172. Kirschbaum C, Hellhammer DH. Salivary cortisol in psychoneuroendocrine research: recent developments and applications. Psychoneuroendocrinology 1994;19:313-33.

173. Whetzel CA, Klein LC. Measuring DHEA-S in saliva: time of day differences and positive correlations between two different types of collection methods. BMC Res Notes 2010;3:204.

174. Beevers CG, Strong DR, Meyer B, et al. Efficiently assessing negative cognition in depression: an item response theory analysis of the Dysfunctional Attitude Scale. Psychol Assess 2007;19:199-209.

175. de Graaf LE, Roelofs J, Huibers MJ. Measuring dysfunctional attitudes in the general population: the dysfunctional attitude Scale (form A) revised. Cognit Ther Res 2009;33:345-55.
176. Shahand S, Santcroos M, van Kampen AHC, et al. A Grid-Enabled Gateway for Biomedical Data Analysis. J Grid Comput 2012;10:725-42.

177. Donders AR, van der Heijden GJ, Stijnen T, et al. Review: a gentle introduction to imputation of missing values. J Clin Epidemiol 2006;59:1087-91.

178. Vaden KI Jr, Gebregziabher M, Kuchinsky SE, et al. Multiple imputation of missing $\mathrm{fMRI}$ data in whole brain analysis. Neurolmage 2012;60:1843-55.

179. Peeters F, Nicolson NA, Berkhof $\mathrm{J}$, et al. Effects of daily events on mood states in major depressive disorder. J Abnorm Psychol 2003;112:203-11.

180. Delespaul PAEGe. Assessing Schizophrenia in Daily Life. The Experience Sampling Method. Maastricht: Maastricht University Press, 1995.

181. Ashburner J, Friston KJ. Voxel-based morphometry--the methods. Neurolmage 2000;11(6 Pt 1):805-21.

182. Ridgway GR, Henley SM, Rohrer JD, et al. Ten simple rules for reporting voxel-based morphometry studies. Neuroimage 2008;40:1429-35.

183. Bach M, Laun FB, Leemans A, et al. Methodological considerations on tract-based spatial statistics (TBSS). Neuroimage 2014:100:358-69.

184. Maninger N, Wolkowitz OM, Reus VI, et al. Neurobiological and neuropsychiatric effects of dehydroepiandrosterone (DHEA) and DHEA sulfate (DHEAS). Front Neuroendocrinol 2009;30:65-91.

185. Mumford JA, Nichols TE. Power calculation for group fMRI studies accounting for arbitrary design and temporal autocorrelation. Neurolmage 2008;39:261-8.

186. Hayasaka S, Peiffer AM, Hugenschmidt CE, et al. Power and sample size calculation for neuroimaging studies by non-central random field theory. Neurolmage 2007;37:721-30.

187. Murphy K, Bodurka J, Bandettini PA. How long to scan? The relationship between $\mathrm{fMRI}$ temporal signal to noise ratio and necessary scan duration. Neuroimage 2007;34:565-74.

188. Siegle GJ, Thompson WK, Collier A, et al. Toward clinically useful neuroimaging in depression treatment: prognostic utility of subgenual cingulate activity for determining depression outcome in cognitive therapy across studies, scanners, and patient characteristics. Arch Gen Psychiatry 2012;69:913-24.

189. Gueorguieva R, Krystal JH. Move over ANOVA: progress in analyzing repeated-measures data and its reflection in papers published in the Archives of General Psychiatry. Arch Gen Psychiatry 2004;61:310-7.

190. Rosenbaum PR, Rubin DB. The Central Role of the Propensity Score in Observational Studies for Causal Effects. Biometrika 1983;70:41-55.

191. Borsboom D, Cramer AO. Network analysis: an integrative approach to the structure of psychopathology. Annu Rev Clin Psychol 2013;9:91-121.

192. van Borkulo CD, Borsboom D, Epskamp S, et al. A new method for constructing networks from binary data. Sci Rep 2014;4:5918.

193. Orrù G, Pettersson-Yeo W, Marquand AF, et al. Using Support Vector Machine to identify imaging biomarkers of neurological and psychiatric disease: a critical review. Neurosci Biobehav Rev 2012;36:1140-52.

194. Epstein RM, Duberstein PR, Feldman MD, et al. "I didn't know what was wrong:" how people with undiagnosed depression recognize, name and explain their distress. J Gen Intern Med 2010;25:954-61.

195. Guo Q, Parlar M, Truong W, et al. The reporting of observational clinical functional magnetic resonance imaging studies: a systematic review. PLOS ONE 2014;9:e94412.

196. Connell L, MacDonald R, McBride T, et al. Observational studies: getting clear about transparency. PLoS Med 2014;11:e1001711.

197. von Elm E, Altman DG, Egger M, et al. Strengthening the Reporting of Observational Studies in Epidemiology (STROBE) statement: guidelines for reporting observational studies. $B M J$ 2007;335:806-8

198. Hrynaszkiewicz I, Norton ML, Vickers AJ, et al. Preparing raw clinical data for publication: guidance for journal editors, authors, and peer reviewers. BMJ 2010;340:c181.

199. Holsboer F, Bender W, Benkert O, et al. Diagnostic value of dexamethasone suppression test in depression. Lancet 1980;2:706.

200. van der Kolk AG, Hendrikse J, Zwanenburg JJ, et al. Clinical applications of 7 T MRI in the brain. Eur J Radiol 2013;82:708-18.

201. Solomon DA, Keller MB, Leon AC, et al. Multiple recurrences of major depressive disorder. Am J Psychiatry 2000;157:229-33.

202. Daumit GL, Dickerson FB, Wang NY, et al. A behavioral weight-loss intervention in persons with serious mental illness. N Engl J Med 2013;368:1594-602. 